\title{
Core-Hole Screening as a Probe for a Metal-to-Nonmetal Transition in Lead Clusters
}

\author{
V. Senz, ${ }^{1, *}$ T. Fischer, ${ }^{2}$ P. Oelßner, ${ }^{1}$ J. Tiggesbäumker, ${ }^{1}$ J. Stanzel, ${ }^{3}$ C. Bostedt, ${ }^{4}$ H. Thomas, ${ }^{4}$ M. Schöffler, ${ }^{5}$ L. Foucar, ${ }^{5}$ \\ M. Martins, ${ }^{6}$ J. Neville, ${ }^{7}$ M. Neeb, ${ }^{3}$ Th. Möller, ${ }^{4}$ W. Wurth, ${ }^{6}$ E. Rühl, ${ }^{8}$ R. Dörner, ${ }^{5}$ H. Schmidt-Böcking, ${ }^{5}$ W. Eberhardt, ${ }^{3}$ \\ G. Ganteför, ${ }^{2}$ R. Treusch, ${ }^{9}$ P. Radcliffe, ${ }^{9}$ and K.-H. Meiwes-Broer ${ }^{1}$ \\ ${ }^{1}$ Institut für Physik, Universität Rostock, 18051 Rostock, Germany \\ ${ }^{2}$ Fachbereich Physik, Universität Konstanz, 78457 Konstanz, Germany \\ ${ }^{3}$ BESSY GmbH, 12489 Berlin, Germany \\ ${ }^{4}$ Institut für Optik und Atomare Physik, Technische Universität Berlin, EW 3-1, 10623 Berlin, Germany \\ ${ }^{5}$ Institut für Kernphysik, Universität Frankfurt, 60438 Frankfurt am Main, Germany \\ ${ }^{6}$ Insitut für Experimentalphysik, Universität Hamburg, 22761 Hamburg, Germany \\ ${ }^{7}$ Department of Chemistry, University of New Brunswick, P.O. Box 45222, Fredericton, NB E3B 6E2, Canada \\ ${ }^{8}$ Insitut für Chemie und Biochemie, Freie Universität Berlin, 14195 Berlin, Germany \\ ${ }^{9}$ Deutsches Elektronen-Synchrotron (DESY), Notkestraße 85, 22603 Hamburg, Germany
}

(Received 22 December 2008; published 3 April 2009)

\begin{abstract}
Metal clusters serve as model systems to study basic problems of electronic correlation. Vacuum ultraviolet light from the free-electron laser FLASH ionizes $5 d$ electrons from mass-separated negatively charged clusters, thus transiently leading to core-ionized neutral systems. Shielding of the core hole affects the electron binding energy. From the strong deviation from expectations of the metallic droplet and jellium models we conclude on reduced electronic shielding once the cluster size falls below about 20 atoms. This suggests a metal-to-nonmetal transition, in agreement with previous local density approximation calculations.
\end{abstract}

DOI: 10.1103/PhysRevLett.102.138303

PACS numbers: $36.20 . \mathrm{Kd}, 36.40 . \mathrm{Ei}, 41.60 . \mathrm{Cr}, 73.22 .-\mathrm{f}$

The properties of matter with physical dimensions on the nanoscale may significantly differ from the corresponding bulk properties. One important feature is the bonding type, which has been shown to undergo metal-to-nonmetal transitions once clusters of alkaline earth elements or mercury fall below a certain size [1-5]. In these divalent materials the situation is comparatively simple as the bonding character appears to be connected to the merging of filled and empty level manifolds. Clusters with open electronic shells where even the valency might change with the number of atoms $N$ have only been treated theoretically. One interesting example in this respect is lead, the valency of which is 2 or 4 . Theoretical atomic structure calculations within density-functional theory predict a metal-to-nonmetal transition at a cluster size of about 20 atoms [6]. A coexistence and competition of two structural growth patterns, a prolate layered structure, which is similar to semiconducting $\mathrm{Si}_{N}$ and $\mathrm{Ge}_{N}$ clusters, and a compact spherical fcc-like structure, is found in the size range between $N=14$ and 22. In this context, a very interesting issue is how a change in bonding is manifested in the electronic correlation. Photoelectron and mass spectroscopy are effective tools for tracing size-dependent bonding changes in the divalent clusters. For lead, however, the richly structured valence photoelectron spectra and the widely scattered electron detachment energies prevent clear insights into a possible band gap closure and the associated change in the bonding character [711]. For tin, the lighter group IV metal with the same valence electron configuration as lead, the band gap closure has recently been observed by means of ultraviolet photoelectron spectroscopy (UPS) at a size of $N=42$ [12].

In this Letter we pursue an alternative approach by using core-hole photoelectron spectroscopy on lead cluster anions $\mathrm{Pb}_{N}^{-}$. Screening in the transiently produced coreionized neutral cluster may serve as a sensor for electron delocalization in the valence band. In fact, VUV and x-ray photoelectron spectroscopy have been widely used on clusters at surfaces [13-16]; for an early review see Ref. [17]. In all cases a characteristic cluster sizedependent shift of the core-level binding energy is observed. For clusters in surface contact many effects contribute to core-level shifts, as there are charge transfer and dielectric screening due to the presence of the surface and structural rearrangements. On isolated and mass-selected systems no inner-shell photoemission has been possible so far due to faint target densities. Nevertheless, there are a few synchrotron-based experiments, among them are corevalence autoionization studied on $\mathrm{Hg}_{N}$ [2], x-ray absorption [18-20] and core-level photoionization on $\mathrm{Pb}_{N}$ without mass selection [21].

In the present approach we start from initially negatively charged clusters. Upon neutralization via photoexcitation of a core level an interesting and new system is created; see Fig. 1. The degree of screening of the localized hole will affect the interaction between the photoelectron and the neutral system left behind. The situation sketched in the figure corresponds to a partially screened core hole, result- 


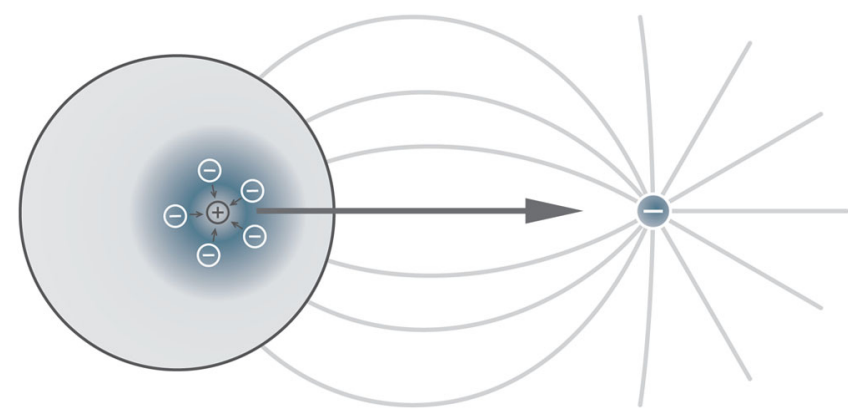

FIG. 1 (color online). A not fully screened core hole attracts electron density and thus reduces the polarizability. As a consequence, the interaction with the photoelectron decreases due to the reduced electrostatic interaction energy.

ing in a deviation from pure metallic behavior. By this, the cluster size-dependent screening can be studied, possibly allowing detection of a change in the bonding character using core-level photoelectron spectroscopy.

The experimental setup, illustrated in Fig. 2, is especially adapted for use at the VUV free-electron laser FLASH and differs in some points from standard pulsed cluster beam experiments. Metal clusters are produced in a laser vaporization cluster source, which is based on known designs, see, e.g., [22]. This type of source generates intense pulsed cluster beams, which is essential for the success of the experiment. Nascent anionic clusters are accelerated and guided by electric fields and massseparated by time of flight. A magnetic bottle electron spectrometer serves to analyze photoelectron energies after irradiation with VUV light pulses. The experiments are performed at FLASH $[23,24]$ beam line BL 1 about $1.5 \mathrm{~m}$ out of the focal point. We deliberately choose an off-focus setup with the accordingly reduced peak intensity in order to illuminate a $1.5 \mathrm{~mm}$ spot of the cluster beam and to avoid nonlinear effects. The photon energy is set to $38 \mathrm{eV}$ $(\sim 32 \mathrm{~nm})$ at a mean pulse energy of about $10 \mu \mathrm{J}$ after beam skimming, corresponding to $1.6 \times 10^{12}$ photons per pulse. The radiation comprised pulse trains consisting of 27 pulses with durations of about $20 \mathrm{fs}$, separated by $10 \mu \mathrm{s}$ and a $5 \mathrm{~Hz}$ repetition rate. We make use of that particular timing pattern by overlapping the FLASH pulse train with the cluster mass spectrum in the time domain, which is illustrated in Fig. 3. Subsequent VUV pulses hit clusters with different, but known, size. Thus each pulse train from FLASH simultaneously creates several photoelectron spectra. In the example shown in Fig. 3, only some of the FLASH pulses hit clusters in between $N=10-50$. By measuring at only a few different FEL time delays, sizeselective photoemission spectra of the whole distribution are recorded. The mass resolution of the apparatus is limited to $m / \Delta m \sim 40$, mainly due to collinear acceleration of cluster ions. Photoelectrons with flight times of up to $600 \mathrm{~ns}$ are detected between two consecutive pulses. Because of the ultralow target density great care has to be

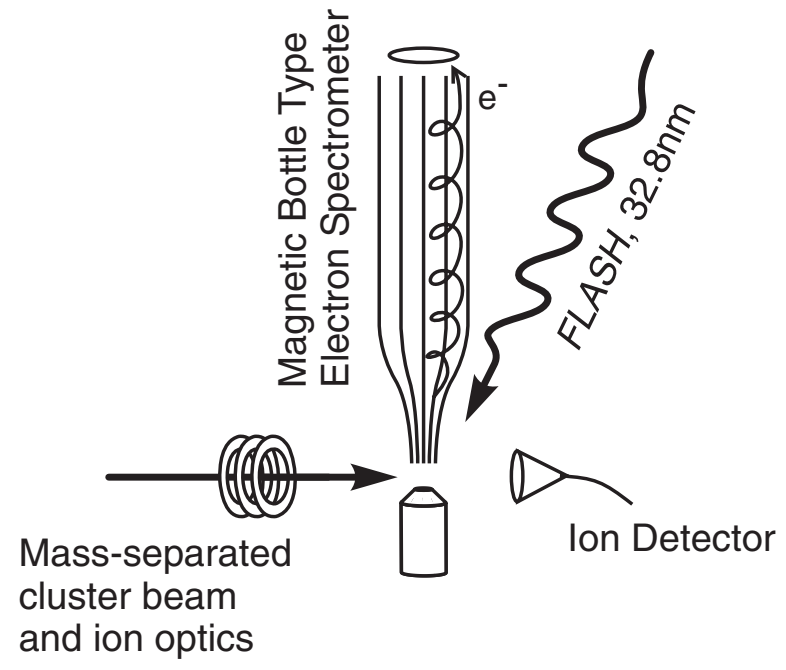

FIG. 2. A metal cluster beam created in a laser vaporization cluster source and time-of-flight mass separated is crossed by the light of the VUV free-electron laser in the interaction region of a magnetic bottle photoelectron spectrometer. Photoelectrons are guided by the magnetic field to the channel-plate detector. Electron time-of-flight spectra are finally converted into binding energy.

taken in order to reduce contributions of photoelectrons from the residual gas and surrounding metal surfaces. The magnetic bottle spectrometer is equipped with a cryogenic shroud cooled with liquid helium. By this, the pressure during operation of the cluster beam can be kept as low as $2 \times 10^{-10}$ mbar.

A set of photoelectron spectra for different cluster sizes is shown in Fig. 4. In spite of ultrahigh vacuum conditions and cryogenic cooling, significant background signal remains beyond $11 \mathrm{eV}$ binding energy. Nevertheless, it is

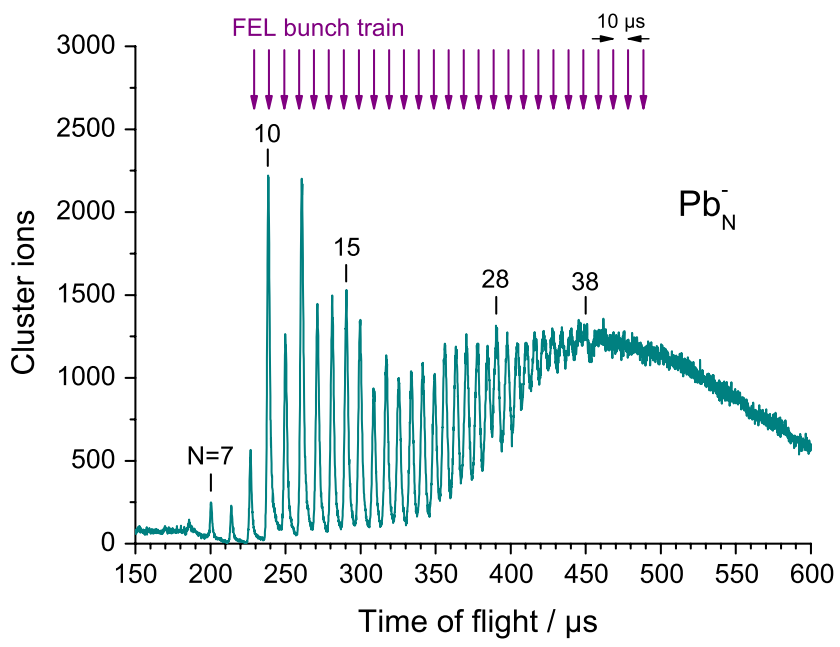

FIG. 3 (color online). Time-of-flight mass spectrum of negatively charged lead clusters. The experiment is synchronized such that the VUV pulses from FLASH interact with clusters of selected size. 
possible to resolve photoemission from the $\mathrm{Pb} 5 d$ core level at around $20 \mathrm{eV}$, as can be seen from the difference spectra on the right-hand side of Fig. 4. The high-energy $5 d_{3 / 2}$ component is clearly discernible for small clusters, but merges with the strong helium photoionization peak at $N>16$ and cannot be resolved anymore $(\operatorname{IE}(\mathrm{He})=$ $24.587 \mathrm{eV}$ [25]). From spectra like those in Fig. 4 lead $5 d_{5 / 2}$ core-level binding energies are extracted and summarized in Fig. 5 with respect to the inverse cluster radius $1 / R$. The radius is calculated from the number of atoms as $R=r_{S} N^{1 / 3}+a_{0}$, with $r_{S}$ the bulk Wigner-Seitz radius and $a_{0}$, the Bohr radius, giving a reasonable approximation of the electron spillout. The experimental data points are compared to calculated electron affinities (EA) within the classical metal sphere approximation [26-30] and local density approximation LDA [8,31], in both cases shifted by the corresponding core-level binding energy. Whereas in conventional anion photoelectron spectroscopy EA refers to the detachment of a valence electron, now the energy scale is pinned to the bulk $5 d_{5 / 2}$ level. Relying on compiled values of $18.1 \mathrm{eV}$ binding energy with respect to the Fermi level and a work function of $4.25 \mathrm{eV}$ [32] results in a $5 d_{5 / 2}$ bulk value of $22.35 \mathrm{eV}$ with respect to the vacuum level. With this energy calibration the size dependence of the core-level binding energy $E_{B}(R)$ can be parameterized for the metal sphere approximation as $E_{B}(R)=E_{B, \infty}-\frac{1}{2} e^{2} / R$; see the solid line in Fig. 5. The LDA calculations, see the dashed curve, include exchange and correlation effects, leading to the expression $E_{B}(R)=$ $E_{B, \infty}-\beta e^{2} / R+O\left(e^{2} / R\right)^{2}$ with $\beta=0.62$. This LDA re-
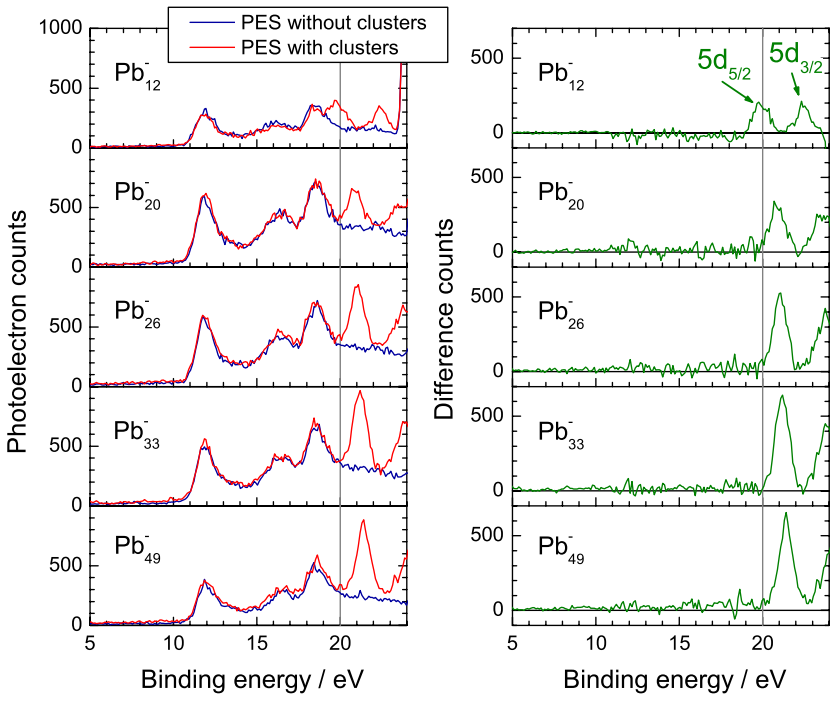

FIG. 4 (color online). Photoelectron spectra of size-selected lead clusters $\mathrm{Pb}_{N}^{-}(N=12-49)$ with and without cluster beam (left) and the corresponding difference spectra (right) after excitation at $38 \mathrm{eV}$ photon energy. A clear shift of the lead $5 d$ core levels is observed, starting from $20 \mathrm{eV}$ (vertical line) at $N=$ 12 towards higher binding energies. (The binding energy is given with respect to the vacuum level.) sult is able to reproduce measured electron affinities for many systems with delocalized electrons, even down to very small clusters [31].

The comparison of the measured $E_{B}$ with the theoretical results shows that for $N>20$ the experimental data follow the behavior of an ideal metallic system, thus hinting at a full screening of the core hole. In other words, for large clusters the $N$-dependent shift of the binding energies agrees with the situation found in valence band photoemission. In that case the classical treatment of the electron detachment (solid line in Fig. 5) is related to a change of the electrostatic charging energy of a metal sphere. However, below $N=20$ the measured core-level binding energies drop faster than theoretically expected. A comparable feature is not clearly apparent in UPS. Obviously, the electrostatic energy of the neutral cluster is significantly reduced, which can be traced back to a loss in electronic screening of the core hole. Indeed, an incompletely screened core hole will attract valence electron density; see Fig. 1. Consequently, there is less polarizability (or image charge build-up) of the neutralized cluster, which will lead to a reduced interaction between photoelectron and cluster. Whereas this model is in qualitative accordance with the observed decrease in binding energy, it has to be checked if the absolute value of the change (about $0.8-1 \mathrm{eV}$ ) is reasonable. For this we consider the atomic core-level binding energy of $\mathrm{Pb}^{-}$(18.6 eV [33]) which ranges about $0.8 \mathrm{eV}$ below the extrapolated theoretical curves in Fig. 5, in accordance with the measurements for the smallest clusters.

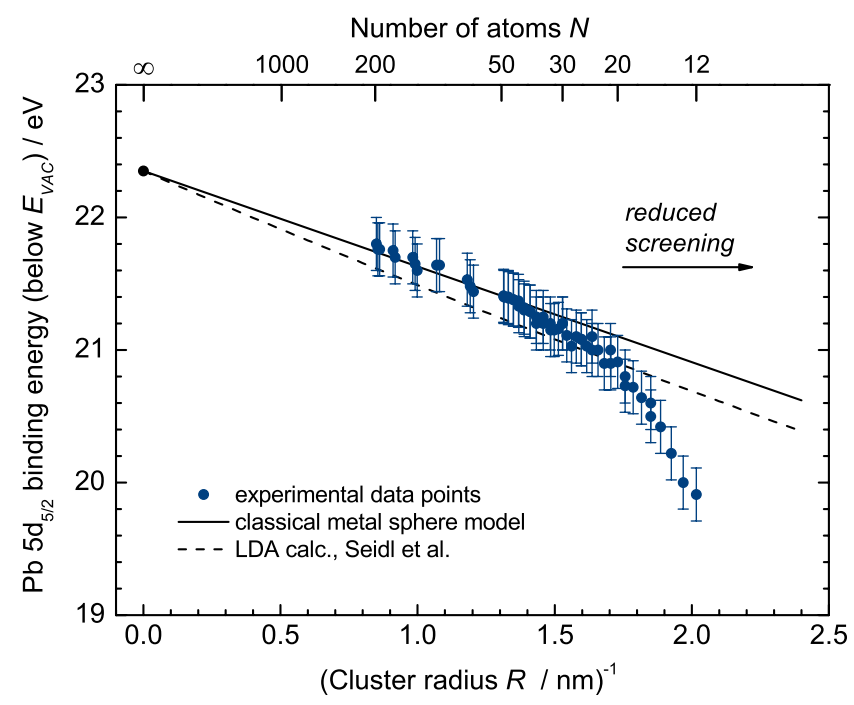

FIG. 5 (color online). Lead $5 d_{5 / 2}$ binding energy versus inverse cluster radius $1 / R(N=12-196)$. For larger cluster sizes the experimental data follow the trend expected from both the classical metal sphere model (solid line) and LDA calculations of Seidl et al. (dashed line) [8,31]. The strong deviation obtained in the low size range indicates a loss in core-hole screening, which leads to a reduced polarizability. This is an indication of a change in the bonding character. 
A loss in screening can be referred to a change in bonding character. In fact, previous DFT calculations on lead clusters found a transition to a nonmetallic state below around $N=20$ [6]. The transition is connected to a structural change where atom-centered compact systems evolve into layered structures with covalent bonding. The calculated density of states hints at a more gradual phase transition instead of a sharp one, which is in agreement with our experimental data.

It should be noted, that the core-ionized neutral cluster is a very short-lived system which will emit a second electron via an Auger process [34]. Still, for the model described here, it is sufficient that the core-hole lifetime exceeds just a few fs, the time needed by the photoelectron to leave the vicinity of the cluster. With higher experimental resolution it might become possible to investigate more details of the electron dynamics, e.g., by analyzing the line profiles. Other effects such as the size-dependent electronic coordination and the contribution of the cluster surface atoms exhibit promising research areas in the future.

In conclusion, core-level photoelectron spectroscopy on size-selected metal cluster anions turns out to be a sensitive probe of core-hole screening and electron delocalization in the valence band of the neutral system. These experiments became possible due to the outstanding high brilliance of the free-electron laser FLASH at DESY in Hamburg. The experiment found a significant deviation of cluster sizedependent core-level binding energies from the predictions of both classical electrodynamics and LDA calculations for ideal metallic systems. The effect is interpreted to be due to a reduced screening of the localized core hole and thus a diminished electrostatic interaction with the photoelectron. This, in turn, can be related to a metal-to-nonmetal transition in agreement with earlier theoretical work.

We gratefully acknowledge experimental support by the whole FLASH team. This work has been supported by the German Federal Ministry of Education and Research under the project number 05KS4HR1-0, the Virtual Institute "Atomic and Cluster Physics with Short Wavelength Radiation from Free-Electron Lasers" of the Helmholtz Society, and the Collaborative Research Center SFB 652 at the University of Rostock.

*volkmar.senz@uni-rostock.de

[1] B. Issendorff and O. Cheshnovsky, Annu. Rev. Phys. Chem. 56, 549 (2005).

[2] C. Bréchignac, M. Broyer, P. Cahuzac, G. Delacretaz, P. Labastie, J. P. Wolf, and L. Wöste, Phys. Rev. Lett. 60, 275 (1988).

[3] R. Busani, M. Folkers, and O. Cheshnovsky, Phys. Rev. Lett. 81, 3836 (1998).

[4] O. C. Thomas, W. Zheng, S. Xu, and K. H. Bowen, Phys. Rev. Lett. 89, 213403 (2002).
[5] T. Diederich, T. Döppner, T. Fennel, J. Tiggesbäumker, and K.-H. Meiwes-Broer, Phys. Rev. A 72, 023203 (2005).

[6] B. Wang, J. Zhao, X. Chen, D. Shi, and G. Wang, Phys. Rev. A 71, 033201 (2005).

[7] G. Ganteför, M. Gausa, K.-H. Meiwes-Broer, and H. Lutz, Z. Phys. D 12, 405 (1989).

[8] C. Lüder and K.-H. Meiwes-Broer, Chem. Phys. Lett. 294, 391 (1998).

[9] B. Wang, L. M. Molina, M. J. Lopez, A. Rubio, J. A. Alonso, and M. J. Stott, Ann. Phys. (Leipzig) 7, 107 (1998).

[10] Y. Negishi, H. Kawamata, A. Nakajima, and K. Kaya, J. Electron Spectrosc. Relat. Phenom. 106, 117 (2000).

[11] L.-F. Cui, X. Huang, L.-M. Wang, J. Li, and L.-S. Wang, J. Phys. Chem. A 110, 10169 (2006).

[12] L.-F. Cui, L.-M. Wang, and L.-S. Wang, J. Chem. Phys. 126, 064505 (2007).

[13] G. K. Wertheim, Z. Phys. D 12, 319 (1989).

[14] W. Eberhardt, P. Fayet, D. M. Cox, Z. Fu, A. Kaldor, R. Sherwood, and D. Sondericker, Phys. Rev. Lett. 64, 780 (1990).

[15] H.-G. Boyen et al., Science 297, 1533 (2002).

[16] H. Hövel, I. Barke, H.-G. Boyen, P. Ziemann, M. G. Garnier, and P. Oelhafen, Phys. Rev. B 70, 045424 (2004).

[17] W. F. Egelhoff, Surf. Sci. Rep. 6, 253 (1987).

[18] P. Piseri et al., New J. Phys. 8, 136 (2006).

[19] J. T. Lau, J. Rittmann, V. Zamudio-Bayer, M. Vogel, K. Hirsch, P. Klar, F. Lofink, T. Möller, and B.v. Issendorff, Phys. Rev. Lett. 101, 153401 (2008).

[20] N. Berrah, R.C. Bilodeau, J.D. Bozek, I. Dumitriu, D. Toffoli, and R. R. Lucchese, Phys. Rev. A 76, 042709 (2007).

[21] S. Peredkov et al., Phys. Rev. B 76, 081402 (2007).

[22] U. Heiz, F. Vanolli, L. Trento, and W.-D. Schneider, Rev. Sci. Instrum. 68, 1986 (1997).

[23] V. Ayvazyan et al., Eur. Phys. J. D 37, 297 (2006).

[24] W. Ackermann et al., Nat. Photon. 1, 336 (2007).

[25] J.E. Sansonetti and W. C. Martin, J. Phys. Chem. Ref. Data 34, 1559 (2005).

[26] G. Makov, A. Nitzan, and L. E. Brus, J. Chem. Phys. 88, 5076 (1988).

[27] J. P. Perdew, Phys. Rev. B 37, 6175 (1988).

[28] M. Seidl and J. P. Perdew, Phys. Rev. B 50, 5744 (1994).

[29] M. Seidl and M. Brack, Ann. Phys. (N.Y.) 245, 275 (1996).

[30] K. Wong, S. Vongehr, and V. V. Kresin, Phys. Rev. B 67, 035406 (2003).

[31] M. Seidl, K.-H. Meiwes-Broer, and M. Brack, J. Chem. Phys. 95, 1295 (1991).

[32] H. B. Michaelson, J. Appl. Phys. 48, 4729 (1977).

[33] K. Hirsch, TU Berlin (private communication), calculation based on R. D. Cowan, The Theory of Atomic Structure and Spectra (University of California Press, Berkeley, CA, 1981).

[34] D. M. P. Holland and K. Codling, J. Phys. B 13, L745 (1980). 